\title{
EFFECT OF HYDRO- ABRASION VERSUS CONVENTIONAL CAVITY PREPARATION ON THE CLINICAL PERFORMANCE OF COMPOSITE RESIN RESTORATION
}

\author{
Laila Taher Kashkosh* , Thuria Mohamed Genaid** and Ali Ibrahem Abdallah**
}

\begin{abstract}
Aim: To evaluate the effect of hydro abrasion versus the conventional drill method of cavity preparation on the clinical performance of composite restoration over one year follow up period.

Materials and methods: Twenty patients with occlusal carious lesions were selected and examined with DIAGNO cam and ICDAS visual criteria to select teeth having ICDAS Code 3 or 4. Forty lesions were randomly divided into two groups $(n=20)$ according to the method of caries removal. Cavities were prepared by the conventional drill method in group I and by hydro-abrasion in group II. Cavities of both groups were restored by self-etch adhesive (Adper Easy Bond) and nano- filled composite (Filtek Z350XT). All restorations were evaluated after 24 hours, after 9 and 12 months according to (USPHS) criteria for retention, marginal adaptation, postoperative sensitivity, marginal discoloration, and secondary caries.
\end{abstract}

Results: Mild, moderate - severe and very severe pain was recorded in group I While in group II, only mild pain was recorded in (40\%) of cases. The statistical analysis of the collected data related to the tested USPHS criteria revealed $100 \%$ Alpha scoring regarding retention, marginal adaptation and secondary caries in both groups throughout the study period. The gap width measured under SEM was found increased sig. by time in both groups. Marginal discoloration was observed in the two groups after nine and 12 months. Post-operative hypersensitivity was found only in group I in $100 \%$ of cases at the base line and was disappeared during the next follow up period.

Conclusion: Hydro-abrasion could be recommended as a preferred method of cavity preparation in shallow to mild carious lesions compared to the conventional cavity preparation method.

KEY WORDS: Dental caries, Cavity preparation, Hydro-abrasion, Composite resin restoration.

\footnotetext{
*Assistant Lecturer of Restorative Dentistry, Faculty of Dentistry, Tanta University, Egypt.

** Professor of Restorative Dentistry, Faculty of Dentistry, Tanta University, Egypt.
} 


\section{INTRODUCTION}

Dental caries is a post-eruptive bacterial infectious disease characterized by a progressive demineralization process that affects the mineralized dental tissues. It is considered to be the most prevalent oral disease worldwide and the main cause of tooth loss among the population ${ }^{1}$. The basic assumption of modern dentistry is the early detection of initial carious lesions. The diagnosis of occlusal carious lesions and the assessment of their severity give clinicians' considerable difficulties resulting from the diversified fissure and pit morphology as well as the widespread use of fluoride compounds ${ }^{2}$.

The International Caries Detection and Assessment System (ICDAS) was developed to standardize visual diagnosis and clinical management of carious lesions. It identifies caries lesions on the basis of their clinical visual appearance; the examination is aided by a ballended explorer and should be carried out on clean and dry teeth ${ }^{3}$. On the other hand, several modern devices are used to detect the presence of early dental caries lesion of which is DIAGNOCam which utilizes near-infrared light that can be used easily in all segments of dentition and was reported as an effective method for the diagnosis of occlusal caries without cavitation in permanent molar teeth ${ }^{4}$.

Major patient reluctance in requesting caries treatment consisted in fear of pain, dental anesthesia, and rotary instruments, especially highspeed hand piece which is associated with some problems including high-pitched noises during use, boneconducted vibration and post-operative dentinal sensitivity, despite its widespread use ${ }^{5}$.

Air abrasion is a technique for cavity preparation which involves the use of aluminum oxide powder, in a fine stream of compressed air. As the particles collide with dentin, the kinetic energy of the particles is released, resulting in fracture of microscopic fragments. In this way, it creates a roughened tooth surface which makes it more conducive to bonding. It produces less heat, sound, and vibration compared to conventional methods leading to fairly pain free dental procedures ${ }^{6}$.

Recently, cutting fluid / air abrasion or Hydroabrasion system is introduced, where a thin, highspeed stream of fluid and microscopic (aluminum oxide) particles removes decay. The fluid acts as a curtain to suppress powder emissions common with the traditional air abrasion units, while simultaneously increasing cutting efficiency by constantly removing debris created during cutting process ${ }^{7}$.

The objective of this study was toreveal the effect of cavity preparation using hydro-abrasion on the clinical performance of composite resin restoration compared to the conventional drill method.

\section{MATERIALS AND METHODS}

The materials used in this study where a nanofilled composite resin and a self-etch adhesive. The material manufacturer, chemical composition, and website of all are illustrated in table (1).

\section{Study design}

The study was conducted as a randomized controlled clinical trial and was carried out at the Restorative Dentistry Department, Faculty of Dentistry, Tanta University.

\section{Ethical considerations}

Approval for this research was obtained from the Research Ethics Committee, Faculty of Dentistry, Tanta University.

\section{Patient selection}

Twenty Patients of both sexes with an average age between (18-25) years, each patient had at least two carious teeth. They were selected from clinic of Restorative Dentistry Department, Faculty of Dentistry, Tanta University to participate in the study, according to exclusion and inclusion criteria.

Scaling and polishing of teeth was performed using rotary brush and low abrasive polishing 
TABLE (1) Materials used in the study:

\begin{tabular}{|c|c|c|c|}
\hline Material & Manufacturer & Composition & Web site \\
\hline $\begin{array}{l}\text { Filtek Z350XT } \\
\text { (nano- filled composite) } \\
\text { (shade A2) }\end{array}$ & 3M ESPE & $\begin{array}{l}\text { Organic matrix: BIS-GMA, UDMA, BIS- } \\
\text { EMA } 6 \text { and small quantities of TEGDMA } \\
\text { Inorganic particle: non agglomerated } \\
\text { nanoparticles of silica } 20 \mathrm{~nm} \text { in size and } \\
\text { Nano agglomerates formed of zirconium } \backslash \\
\text { silica particles ranging from } 0.6 \text { to } 1 . \mathrm{Mm} \text { in } \\
\text { size ( } 78.5 \text { Weight } \% \text {.) }\end{array}$ & \multirow[t]{2}{*}{ WWW.3M.com } \\
\hline $\begin{array}{l}\text { Adper }^{\mathrm{Tm}} \\
\text { Easy Bond } \\
\text { Self-Etch Adhesive }\end{array}$ & 3M ESPE & $\begin{array}{l}\text { Methacrylated phosphoric esters, } \\
\text { dimethacrylates, 2-HEMA, polyalkenoid } \\
\text { acid copolymer, colloidal silica, ethanol, } \\
\text { water, photoinitiator. }\end{array}$ & \\
\hline
\end{tabular}

BIS-GMA: BIS-phenol A Glycidyl Meth-Acrylate

UDMA: Urethane Di-Meth-Acrylate

BIS-EMA: BIS-phenol Ethoxylated Meth-Acrylate

TEGDMA: Tri-Ethylene Glycol Di-Meth-Acrylate

HEMA: Hydroxy Ethyl Meth-Acrylate

paste (Dharma Quartz prophylaxis paste, USA) to remove calculus and any stains that may affect caries diagnosis and/or composite shade selection. The teeth were partially isolated using cotton rolls and air dried for 5 seconds and examined using a dental mirror, a blunt calibrated periodontal probe, compressed air, and the light source of the dental unit. Caries assessment status of the occlusal surface of selected teeth was performed according to International Caries Detection Assessment System (ICDAS) visual criteria by two examiners ${ }^{8}$.

Teeth with ICDAS Code 3 (localized enamel breakdown due to caries with no visible dentin or underlying shadow) and Code 4 (underlying dark shadow from dentin with or without localized enamel breakdown) were only used in this study. Maintaining teeth isolation, the visual assessment was confirmed by DIAGNOcam (KaVo Dental Gmbh, Biberach, Germany) ${ }^{9}$.

The selected teeth were isolated with a rubber dam (Coltene Whale dent India). After that, a single layer of a separation agent (AD-Q131 separating agent, alpha dental Equipment Co, China) was applied on the occlusal surface before making an occlusal stamp to facilitate its removal. Flowable composite resin (Nexcomp Flow; MetaBiomed, Chungbuk, Korea) was applied, covering the entire occlusal surface, and going a little over the cusps. A tip of a micro brush was cut and immersed into the flowable composite applied on the occlusal surface before being polymerized using light curing device (Blue phase $\mathrm{N}$ Ivoclar vivadent) for 20 seconds acting as a handle to the occlusal stamp.

\section{Grouping of selected teeth}

A total of forty posterior teeth were used in this study that was randomly divided in two groups according to the method of caries removal as follow:

Group I: The carious lesions were removed by the conventional drill method using no. 245 carbide burs (Midwest, Dentsply) and high speed contra angle hand piece (NSk, JAPAN) with adequate water coolant system. The burs were replaced with new ones after every five preparations. Cavity designs were restricted to remove caries with no undercuts, no beveling ${ }^{10}$. 
Group II: The carious lesions were removed by Aquacut Quattro air abrasion unit (Aquacut Quattro, Velopex, London, UK air abrasion system) which uses a gamma irradiated aluminum oxide particle as abrasives (29 micron), cutting fluid and a handpiece of $0.6 \mathrm{~mm}$ diameter tip.

\section{Restorative procedure:}

The prepared cavities were rinsed with water, dried and carefully isolated. Self-etch adhesive (Adper ${ }^{\mathrm{Tm}}$ Easy Bond) and composite material (Filtek Z350XT nano- filled composite- shade A2) were applied following the manufacturer's instructions as follows:

A layer of self-etch adhesive was applied to all cavity surfaces and brushed for 20 seconds using disposable micro brush followed by gentle air dispersion and light curing for 10 seconds using light curing device.

Cavities of both groups were incrementally restored by the composite material and each increment was light cured for 20 seconds. On the last composite layer, a piece of Teflon tape (PTFE Tape, China), was applied on the occlusal surface followed by adaptation of the occlusal stamp. Excess material and the Teflon were then removed, composite was cured, and the occlusion was checked by articulating paper (Tangshan UMG Medical Instrument Co.). The restorations were then finished with finishing points (DENTSPLY, Australia) and polished following the manufacturers' instructions using polishing paste (EZ-SHINE, EZ-PAC, Egypt) and golden brush (KaVo Kerr Composite Polishing Brush, China).

\section{Evaluation procedures}

\section{Clinical evaluation}

The date of base line and subsequent follow up visits were recorded in the patient files. The patients were examined at the dental clinic and digital photographs were taken for future reference and documentation.
Patient anxiety was recorded during cavity preparation procedure according to the visual analogue scale (VAS). Subjects were trained on how to use the scale at the screening visit. They were asked to rate the intensity of their response to pain by marking off the line such that it corresponds to the severity of perceived pain. The pain intensity was shown as an absolute value or as a percentage of the maximum using the $100-\mathrm{mm}$ line ranging from (0-no pain to 100 -worst pain imaginable) ${ }^{11}$.

To evaluate any possible postoperative sensitivity, the patients were verbally questioned regarding the following aspects: sensitivity to cold and/or hot, spontaneous pain either prolonged or not and pain during mastication and sensitivity from other stimuli ${ }^{12}$.

The restored teeth were subjected to a clinical follow up schedule representing three follow up periods (24 hours after restoration placement (baseline), 9 months and 12 months). The data of the tested criteria were collected at these observation times.

Two examiners not involved in the placement of the restorations were calibrated to evaluate the restoration according to the modified (USPHS) criteria for marginal adaptation, marginal discoloration, secondary caries, retention, and postoperative sensitivity. The evaluators were not informed or unaware of the materials or grouping used in this study. Evaluation was done under a dental operating light, using flat surfaced mouth mirrors and dental explorers ${ }^{13}$.

Scores were recorded as follows: alpha represents the ideal clinical situation; Bravo means clinically acceptable; Charlie is considered a clinically unacceptable situation where the restoration has to be replaced ${ }^{14}$.

An evaluation sheet was used to record the patient scores at each follow up visit. In case of discrepancies in scoring, restorations were 
reevaluated and a consensus was reached. A clinical photograph was made at each follow-up for further comparison ${ }^{15}$.

\section{Scanning Electron Microscope (SEM) evaluation of marginal adaptation:}

The marginal seal clinical results were confirmed by SEM examination of an inverse replica of randomly selected 15 restorations for each tested group at the different follow up periods. Replicas were prepared utilizing a silicone impression material (Aquasil Ultra LV. Dentsply) that examined under SEM (JEOL JXA-840, a scanning microscope, USA).

These replicas were mounted on custom-made aluminum stubs, gold-sputtered, and examined under SEM initially at magnifications up to X12 \& X13 (For the overall view of the restoration) followed by a higher magnification X 250 (For the entire restoration interfaces).

SEM images were taken of the occlusal restoration interfaces that investigated for detection of marginal gaps. The mean marginal gap width calculated by selecting 8 different points ( 3 buccal, 3 lingual, 1 mesial, 1 distal) and was recorded for each examined restoration ${ }^{16}$.

\section{Statistical analysis:}

The collected data along all the evaluation periods were collected, tabulated and statistically analyzed using software statistical package for social science (SPSS) version 26.

\section{RESULTS}

The anxiety and operative pain (VAS) was recorded during cavity preparation in both groups. As in table (1) revealed that, in group I, $(0 \%)$ of patients recorded no pain, (10\%) mild pain, (35\%) moderate- severe pain and $(20 \%)$ very severe pain. However, in group II (60\%) recorded no pain, (40\%) mild pain and $(0 \%)$ were found for moderate, severe, and very severe pain. Chi-Square test revealed statistically significant difference between the groups $(\mathrm{p}=0.000)$.

The tested criteria (restoration retention, marginal adaptation, marginal seal, postoperative hypersensitivity, marginal discoloration, and secondary caries) were evaluated according to the modified USPHS criteria. This was conducted at the base line ( 24 hour), 9 months and 12 months' intervals. A hundred \% recall rate was recorded at all evaluation periods.

The retention rate of restorations in both tested groups at the different evaluation periods was presented in table (2). As seen a $100 \%$ retention rate was found in both groups in different follow up periods.

Since marginal adaptation was assumed to affect the durability of restorations so it was evaluated more accurately using SEM to detect the marginal seal of different restorations (15 representative samples in each group) at different follow up periods. Data was

TABLE (1): Percentages of the recorded pain (VAS) during cavity preparation

\begin{tabular}{|c|c|c|c|c|c|c|c|c|c|c|c|c|c|c|}
\hline Severity of pain & \multicolumn{2}{|c|}{ No pain } & \multicolumn{2}{|c|}{ Mild } & \multicolumn{2}{c|}{ Moderate } & \multicolumn{2}{c|}{ Severe } & \multicolumn{2}{c|}{$\begin{array}{c}\text { Very } \\
\text { severe }\end{array}$} & \multicolumn{2}{|c|}{ Total } & \multicolumn{2}{c|}{ Chi- Square Test } \\
\hline Groups & N & $\%$ & N & $\%$ & N & $\%$ & N & $\%$ & N & $\%$ & N & $\%$ & $\chi 2$ & p-value \\
\hline $\begin{array}{c}\text { Group I Cavities prepared by } \\
\text { conventional drill method }\end{array}$ & $\mathbf{0}$ & $\mathbf{0}$ & $\mathbf{2}$ & $\mathbf{1 0}$ & $\mathbf{7}$ & $\mathbf{3 5}$ & $\mathbf{7}$ & $\mathbf{3 5}$ & $\mathbf{4}$ & $\mathbf{2 0}$ & $\mathbf{2 0}$ & $\mathbf{1 0 0}$ & & \\
\hline $\begin{array}{c}\text { Group II Cavities prepared } \\
\text { by hydro-abrasion method }\end{array}$ & $\mathbf{1 2}$ & $\mathbf{6 0}$ & $\mathbf{8}$ & $\mathbf{4 0}$ & $\mathbf{0}$ & $\mathbf{0}$ & $\mathbf{0}$ & $\mathbf{0}$ & $\mathbf{0}$ & $\mathbf{0}$ & $\mathbf{2 0}$ & $\mathbf{1 0 0}$ & & \\
\hline
\end{tabular}


TABLE (V-2): The $\%$ of retention rate scores of restorations in both groups at different follow up periods:

\begin{tabular}{|c|c|c|c|c|c|c|c|}
\hline \multicolumn{9}{|c|}{ Retention rate } \\
\hline \multirow{2}{*}{$\begin{array}{c}\text { Time /Score } \\
\text { Groups }\end{array}$} & \multirow{2}{*}{ Score } & \multicolumn{2}{|c|}{ Baseline } & \multicolumn{2}{c|}{$\mathbf{9}$ Months } & \multicolumn{2}{|c|}{$\mathbf{1 2}$ Months } \\
\cline { 3 - 8 } & & $\mathbf{N}$ & $\mathbf{( \% )}$ & $\mathbf{N}$ & $\mathbf{( \% )}$ & $\mathbf{N}$ & $\mathbf{( \% )}$ \\
\hline $\begin{array}{c}\text { Group I } \\
\text { Cavities prepared by conventional drill } \\
\text { method. }\end{array}$ & Alpha & $\mathbf{2 0}$ & $\mathbf{1 0 0}$ & $\mathbf{2 0}$ & $\mathbf{1 0 0}$ & $\mathbf{2 0}$ & $\mathbf{1 0 0}$ \\
\cline { 3 - 9 } & Charlie & $\mathbf{0}$ & $\mathbf{0}$ & $\mathbf{0}$ & $\mathbf{0}$ & $\mathbf{0}$ & $\mathbf{0}$ \\
\hline $\begin{array}{c}\text { Group II } \\
\text { Cavities prepared by hydro-abrasion method }\end{array}$ & Alpha & $\mathbf{2 0}$ & $\mathbf{1 0 0}$ & $\mathbf{2 0}$ & $\mathbf{1 0 0}$ & $\mathbf{2 0}$ & $\mathbf{1 0 0}$ \\
\cline { 2 - 8 } & Charlie & $\mathbf{0}$ & $\mathbf{0}$ & $\mathbf{0}$ & $\mathbf{0}$ & $\mathbf{0}$ & $\mathbf{0}$ \\
\hline
\end{tabular}

Regarding the marginal adaptation of the restorations, table (3) revealed a 100\% Alpha scoring (complete marginal adaptation) in both groups throughout the study period.

TABLE (3): The \% of marginal adaptation scores of restorations in both groups at different follow up periods:

\begin{tabular}{|c|c|c|c|c|c|c|c|}
\hline \multicolumn{8}{|c|}{ Marginal adaptation } \\
\hline \multirow{2}{*}{$\begin{array}{l}\text { Time /Score } \\
\text { Groups }\end{array}$} & \multirow{2}{*}{ Score } & \multicolumn{2}{|c|}{ Baseline } & \multicolumn{2}{|c|}{9 Months } & \multicolumn{2}{|c|}{12 Months } \\
\hline & & $\mathbf{N}$ & $(\%)$ & $\mathbf{N}$ & $(\%)$ & $\mathbf{N}$ & $(\%)$ \\
\hline \multirow{3}{*}{$\begin{array}{c}\text { Group I } \\
\text { Cavities prepared by conventional drill method. }\end{array}$} & Alpha & 20 & 100 & 20 & 100 & 20 & 100 \\
\hline & Bravo & $\mathbf{0}$ & $\mathbf{0}$ & $\mathbf{0}$ & $\mathbf{0}$ & $\mathbf{0}$ & $\mathbf{0}$ \\
\hline & Charlie & $\mathbf{0}$ & $\mathbf{0}$ & $\mathbf{0}$ & $\mathbf{0}$ & $\mathbf{0}$ & $\mathbf{0}$ \\
\hline \multirow{3}{*}{$\begin{array}{c}\text { Group II } \\
\text { Cavities prepared by hydro-abrasion method. }\end{array}$} & Alpha & 20 & 100 & 20 & 100 & 20 & 100 \\
\hline & Bravo & $\mathbf{0}$ & $\mathbf{0}$ & $\mathbf{0}$ & $\mathbf{0}$ & $\mathbf{0}$ & $\mathbf{0}$ \\
\hline & Charlie & $\mathbf{0}$ & $\mathbf{0}$ & $\mathbf{0}$ & $\mathbf{0}$ & $\mathbf{0}$ & $\mathbf{0}$ \\
\hline
\end{tabular}

presented in table (4). At base line, mean value of gap width in restorations of both tested groups was zero denoting complete marginal seal of all tested restorations. After 9 months, a mean value of 3.18 $\mu \mathrm{m}$ was recorded in group I (cavities prepared by conventional drill method). While a gap width mean value of $2.23 \mu \mathrm{m}$ was recorded for group II (cavities (prepared by hydro-abrasion unit). After 12 months, there was further marginal deterioration where a high value of gap width $(8.33 \mu \mathrm{m})$ was recorded in group I while in group II a lower value was still recorded $(4.88 \mu \mathrm{m})$.

Repeated measure ANOVA and multiple comparisons (Tukey test) were used to compare between durations in each group. Independent t-test was used to compare groups in each duration as in table (4).
Regarding the effect of time on marginal seal, ANOVA test revealed statistically highly significant difference between different evaluation periods in both groups $(\mathrm{p}=0.000)$. While $\mathrm{t}$ test revealed no statistically significant difference between the two groups after 9 month follow-up period ( $\mathrm{p}=0.260$ ). However, after 12 months follow up period, marginal seal recorded a statistically significant difference $(p=0.002)$ between two groups where the gap width in in group I $(8.33 \mu \mathrm{m})$ was significantly higher than in group II $(4.88 \mu \mathrm{m})$.

Figures (1-6) showing representative SEM photomicrographs of resin replica of restorations of both groups at the different evaluation periods.

Furthermore pair wise Tukey test was performed to compare between the different evaluation periods 
TABLE (4): Statistical analysis of the mean marginal gap width values of restorations at different follow up periods:

\begin{tabular}{|c|c|c|c|c|c|}
\hline \multicolumn{6}{|c|}{ Marginal seal } \\
\hline \multirow{2}{*}{ Groups } & Baseline & 9 Months & 12 Months & \multicolumn{2}{|c|}{ ANOVA Test } \\
\hline & Mean \pm S.D & Mean \pm S.D & Mean \pm S.D & $\mathrm{F}$ & p-value \\
\hline $\begin{array}{c}\text { Group I } \\
\text { Cavities prepared by conventional drill method. }\end{array}$ & 0 & $3.18 \pm 2.49$ & $8.33 \pm 2.75$ & 93.107 & $0.000 * * *$ \\
\hline $\begin{array}{c}\text { Group II } \\
\text { Cavities prepared by hydro-abrasion method. }\end{array}$ & 0 & $2.23 \pm 2.01$ & $4.88 \pm 2.80$ & 39.225 & $0.000 * *$ \\
\hline $\mathrm{t}$ & ------ & 1.150 & 3.405 & \multirow{2}{*}{----- } & \multirow{2}{*}{---------- } \\
\hline$p$-value & ------ & 0.260 & $0.002 *$ & & \\
\hline
\end{tabular}

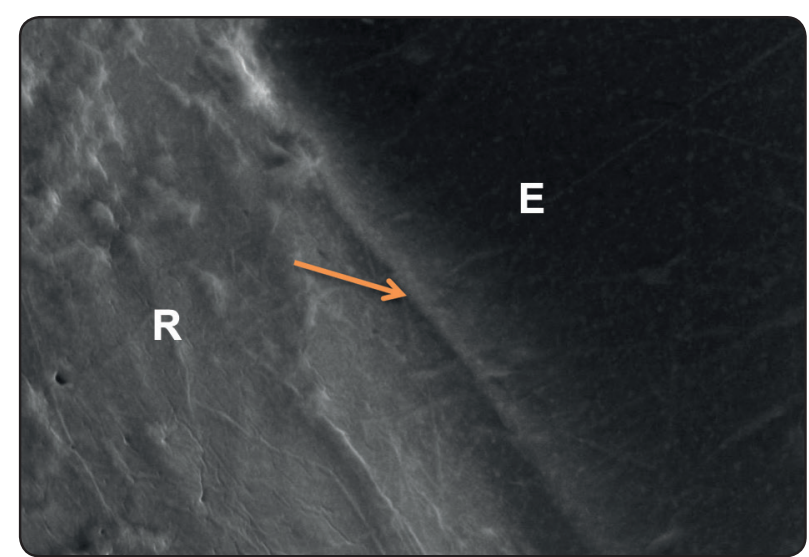

Fig. (1): SEM image at magnification x250 of inverse replica of (group I) restoration at base line evaluation showing sealed margin interface between the restoration $(\mathrm{R})$ \& tooth enamel $(\mathrm{E})$. between the restoration $(\mathrm{R})$ \& tooth enamel (E).

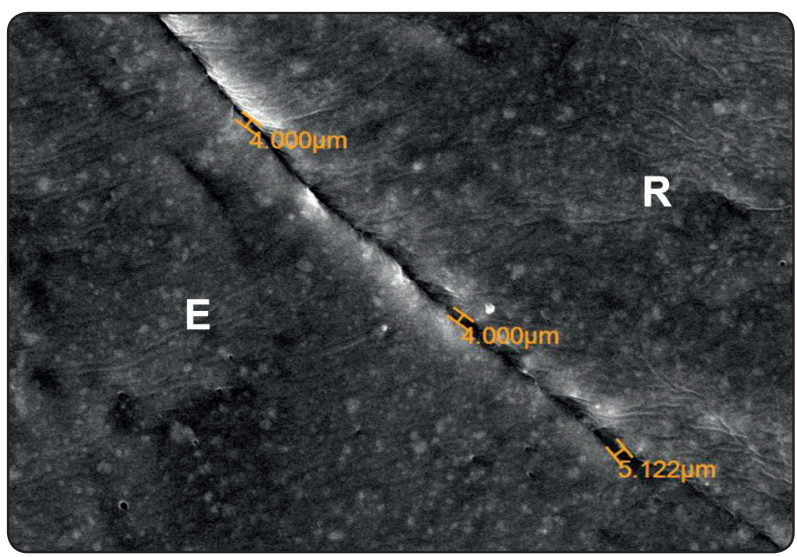

Fig. (3): SEM image at magnification x250 of inverse replica of (group I) restoration after one year follow up period showing micro gaps at interface between the restoration (R) \& tooth enamel (E).

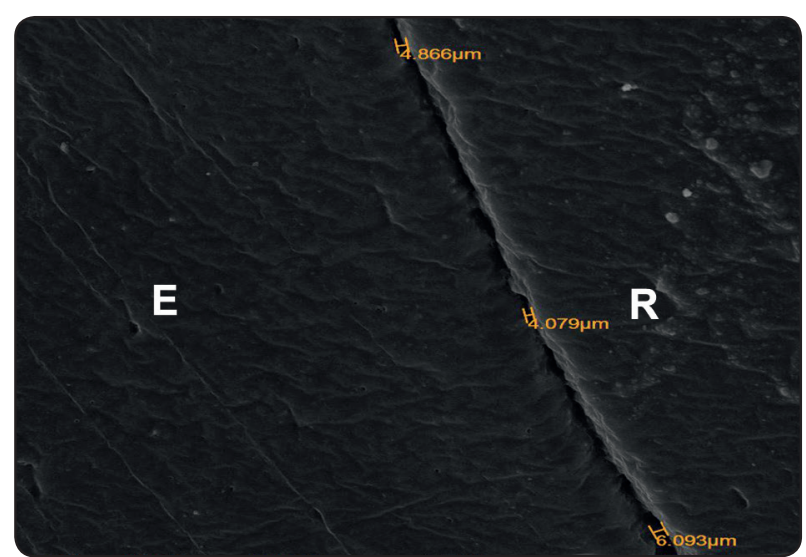

Fig. (2): SEM image at magnification x 250 of inverse replica of (group I) restoration after nine months follow up period showing micro gaps at interface between the restoration (R) \& tooth enamel (E).

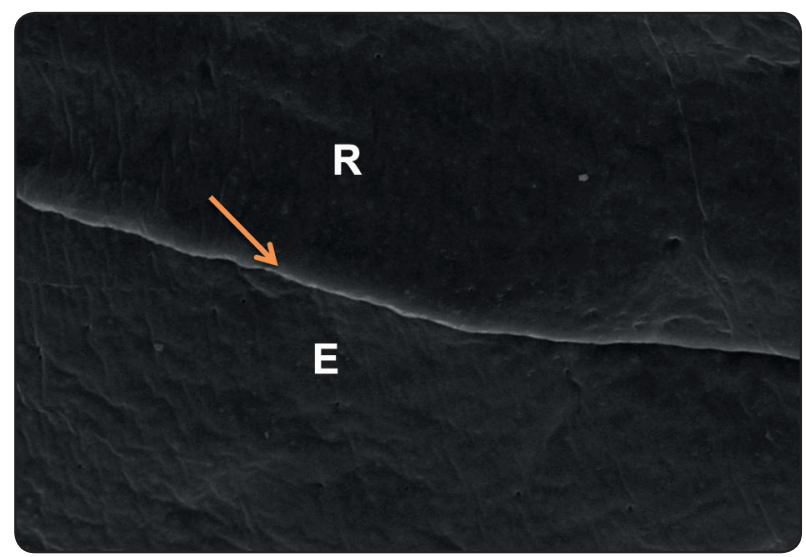

Fig. (4): SEM image at magnification x 250 of inverse replica (group II) restoration at the base line evaluation showing sealed margin interface between the restoration $(\mathrm{R})$ \& tooth enamel (E). 


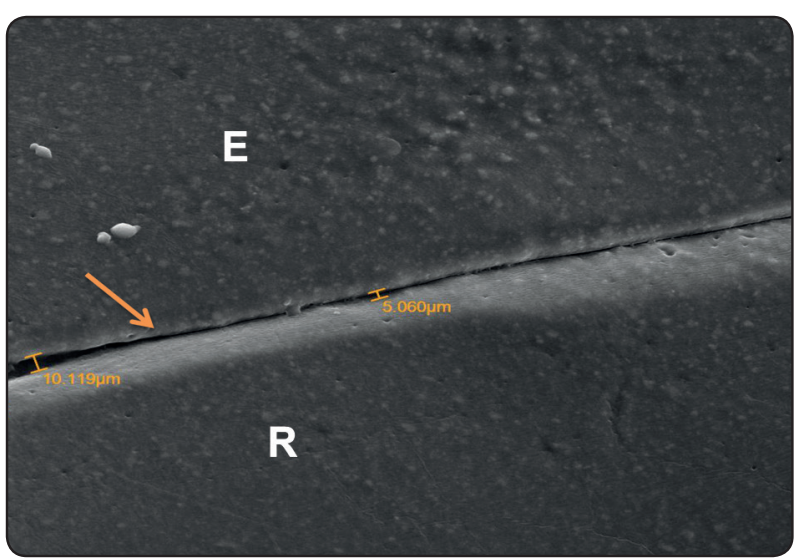

Fig. (5): SEM image at magnification x 250 of inverse replica of (group II) restoration after nine months follow up period showing micro gaps at interface between the restoration $(\mathrm{R})$ \& tooth enamel $(\mathrm{E})$

in each group table (5). It revealed a statistically highly significant difference between the base line versus both $9 \& 12$ month as well as between 9 versus 12 month evaluation periods in group I $(\mathrm{p}=0.000)$. Similar findings was found in group II $(p=0.001 \& 0.000)$ respectively.

The collected data regarding postoperative hypersensitivity for both tested groups at different follow up periods was presented in table (6). As seen in the table, Bravo score (sensitivity with diminishing intensity) was found only in group I (cavities prepared by conventional drill method) in $100 \%$ of cases at the base line and was disappeared

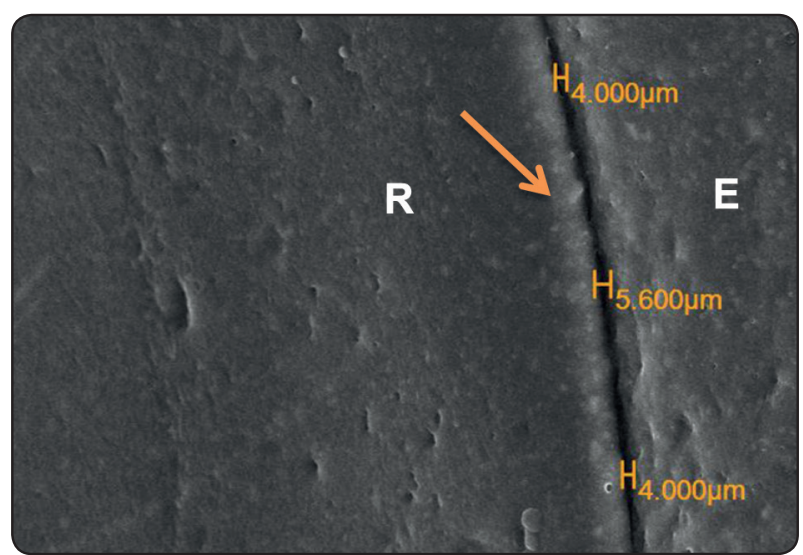

Fig. (6): SEM image at magnification x250 of inverse replica of (group II) restoration after one year follow up period showing micro gaps at interface between the restoration (R) \& enamel tooth structure (E).

completely during the next follow up period. While Alpha scores (no postoperative hypersensitivity) was recorded in group II (cavities prepared by hydro-abrasion) at any of the evaluation periods.

Chi-Square test revealed a statistically highly significant difference between the different evaluation periods in group I $(p=0.000)$. In addition, the test revealed a statistically highly significant difference between the two groups at base line observation ( $\mathrm{p}=0.000)$.

Concerning marginal discoloration, the collected data is presented table (7). The two groups revealed

TABLE (5): Pairwise Tukey test of the mean marginal gap width values in both groups at the different follow up periods:

\begin{tabular}{|c|c|c|c|}
\hline Groups & Duration & Baseline & 9 Months \\
\hline \multirow{3}{*}{$\begin{array}{l}\text { Group I } \\
\text { Cavities prepared by conventional drill } \\
\text { method. }\end{array}$} & Baseline & ---------- & --------- \\
\hline & 9 Months & $0.000 * *$ & ---------- \\
\hline & 12 Months & $0.000 * *$ & $0.000 * *$ \\
\hline \multirow{3}{*}{$\begin{array}{l}\text { Group II } \\
\text { Cavities prepared by hydro-abrasion } \\
\text { method. }\end{array}$} & Baseline & ---------- & ----------- \\
\hline & 9 Months & $0.001 *$ & --------- \\
\hline & 12 Months & $0.000 * *$ & $0.000 * *$ \\
\hline
\end{tabular}


(100\%) Alpha scores after 24 hours. At nine months and 12 months group I (cavities prepared by conventional drill method) recorded (65\%) Alpha scores, (20\%) Bravo scores and (15\%) Charlie scores. While after nine months and 12 months, group II (cavities prepared by hydro-abrasion) recorded (85\%) Alpha scores, (5\%) Bravo scores and $(10 \%)$ Charlie scores. Regarding the effect of time on the marginal discoloration Chi-Square test revealed a statistically highly significant difference among the evaluation periods in both group $(\mathrm{P}=$ $0.000)$. However, the test revealed no statistically significant difference between the two groups at either 9 or 12 recall periods $(p=0.282)$.

The collected data revealed no secondary caries at any evaluation period in the two groups as shown in table (8). All restorations recorded Alpha score; thus no statistical analysis was performed.

TABLE (6): Statistical analysis of the post-operative hypersensitivity scores of both groups at different follow up periods:

\begin{tabular}{|c|c|c|c|c|c|c|c|c|c|}
\hline \multicolumn{10}{|c|}{ Postoperative sensitivity } \\
\hline \multirow{2}{*}{$\begin{array}{l}\text { Time /Score } \\
\text { Groups }\end{array}$} & \multirow{2}{*}{ Score } & \multicolumn{2}{|c|}{ Baseline } & \multicolumn{2}{|c|}{9 Months } & \multicolumn{2}{|c|}{12 Months } & \multicolumn{2}{|c|}{ Chi- Square Test } \\
\hline & & $\mathrm{N}$ & $(\%)$ & $\mathrm{N}$ & $(\%)$ & $\mathrm{N}$ & $(\%)$ & $\chi^{2}$ & p-value \\
\hline \multirow{3}{*}{$\begin{array}{c}\text { Group I } \\
\text { Cavities prepared by conventional drill } \\
\text { method }\end{array}$} & Alpha & 0 & 0 & 20 & 100 & 20 & 100 & \multirow{3}{*}{60.000} & \multirow{3}{*}{$0.000 * *$} \\
\hline & Bravo & 20 & 100 & 0 & 0 & 0 & 0 & & \\
\hline & Charlie & 0 & 0 & 0 & 0 & 0 & 0 & & \\
\hline \multirow{3}{*}{$\begin{array}{c}\text { Group II } \\
\text { Cavities prepared by hydro-abrasion t. }\end{array}$} & Alpha & 20 & 100 & 20 & 100 & 20 & 100 & \multirow{3}{*}{------ } & \multirow{3}{*}{------ } \\
\hline & Bravo & 0 & 0 & 0 & 0 & 0 & 0 & & \\
\hline & Charlie & 0 & 0 & 0 & 0 & 0 & 0 & & \\
\hline \multirow{2}{*}{ Chi- Square Test } & $\chi^{2}$ & \multicolumn{2}{|c|}{40.001} & \multirow{2}{*}{\multicolumn{2}{|c|}{------ }} & \multirow{2}{*}{\multicolumn{2}{|c|}{------ }} & \multirow{2}{*}{------ } & \multirow{2}{*}{-------. } \\
\hline & p-value & \multicolumn{2}{|c|}{$0.000 * *$} & & & & & & \\
\hline
\end{tabular}

TABLE (7): Statistical analysis of the marginal discoloration scores of restorations at different follow up periods:

\begin{tabular}{|c|c|c|c|c|c|c|c|c|c|}
\hline \multicolumn{10}{|c|}{ Marginal discoloration } \\
\hline \multirow{2}{*}{$\begin{array}{c}\text { Time/Score } \\
\text { Groups }\end{array}$} & \multirow{2}{*}{ Score } & \multicolumn{2}{|c|}{ Baseline } & \multicolumn{2}{|c|}{9 Months } & \multicolumn{2}{|c|}{12 Months } & \multicolumn{2}{|c|}{ Chi- Square Test } \\
\hline & & $\mathrm{N}$ & $(\%)$ & $\mathrm{N}$ & $\%)$ & $\mathrm{N}$ & $\%)$ & $\chi^{2}$ & p-value \\
\hline \multirow{3}{*}{$\begin{array}{c}\text { Group I } \\
\text { Cavities prepared by conventional drill } \\
\text { method. }\end{array}$} & Alpha & 20 & 100 & 13 & 65 & 13 & 65 & \multirow{3}{*}{34.286} & \multirow{3}{*}{$0.000 * *$} \\
\hline & Bravo & 0 & 0 & 4 & 20 & 4 & 20 & & \\
\hline & Charlie & 0 & 0 & 3 & 15 & 3 & 15 & & \\
\hline \multirow{3}{*}{$\begin{array}{l}\text { Group II } \\
\text { Cavities prepared by hydro-abrasion } \\
\text { method. }\end{array}$} & Alpha & 20 & 100 & 17 & 85 & 17 & 85 & \multirow{3}{*}{51.818} & \multirow{3}{*}{$0.000 * *$} \\
\hline & Bravo & 0 & 0 & 1 & 5 & 1 & 5 & & \\
\hline & Charlie & 0 & 0 & 2 & 10 & 2 & 10 & & \\
\hline \multirow{2}{*}{ Chi- Square Test } & $\chi^{2}$ & \multirow{2}{*}{\multicolumn{2}{|c|}{------ }} & \multicolumn{2}{|c|}{2.533} & \multicolumn{2}{|c|}{2.533} & \multirow{2}{*}{------ } & \multirow{2}{*}{-------- } \\
\hline & p-value & & & \multicolumn{2}{|c|}{0.282} & \multicolumn{2}{|c|}{0.282} & & \\
\hline
\end{tabular}


Spearman's correlation test was performed between the related tested criteria in each group to study their statistical relationship. The Spearman correlation coefficients (r) ranges from $(-1)$ to $(+1)$, where $(+)$ values indicate positive relation while $(-)$ values indicates negative relation. The relation was considered significant difference at $\mathrm{P}$-value $<0.05$ and highly significance if $<0.001$. The recorded (r) values in this study were all $(+)$ indicating positive relations between the compared criteria.
Regarding the relationship between marginal adaptation and marginal discoloration, table (9) showed no statistically significant relation $(\mathrm{P}=$ 0.081 neither after 9 nor 12 months follow up period in group I (cavities prepared by conventional drill method). On the other hand, in group II (cavities prepared by hydro-abrasion) there was a statistically significant relation between both criteria $(\mathrm{P}=0.008)$ after $9 \& 12$ months.

TABLE (8): Statistical analysis of the secondary caries scores of restorations at different follow up periods:

\begin{tabular}{|c|c|c|c|c|c|c|c|}
\hline \multicolumn{3}{|c|}{ Secondary caries } \\
\hline \multirow{2}{*}{$\begin{array}{c}\text { Time/Score } \\
\text { Groups }\end{array}$} & Score & \multicolumn{2}{|c|}{ Baseline } & \multicolumn{2}{|c|}{9 Months } & \multicolumn{2}{c|}{12 Months } \\
\cline { 3 - 8 } & & $\mathrm{N}$ & $(\%)$ & $\mathrm{N}$ & $(\%)$ & $\mathrm{N}$ & $(\%)$ \\
\hline $\begin{array}{c}\text { Group I } \\
\text { Cavities prepared by conventional drill } \\
\text { method. }\end{array}$ & Alpha & 20 & $100 \%$ & 20 & $100 \%$ & 20 & 100 \\
\cline { 2 - 9 } $\begin{array}{c}\text { Group II } \\
\text { Cavities prepared by hydro-abrasion method. }\end{array}$ & Charlie & 0 & $0 \%$ & 0 & 0 & 0 & 0 \\
\cline { 2 - 9 } & Charlie & 0 & $0 \%$ & 0 & 0 & 0 & 0 \\
\hline
\end{tabular}

TABLE (9): The relationship between marginal adaptation versus marginal discoloration of the two groups at different follow up periods:

\begin{tabular}{|c|c|c|c|c|c|c|}
\hline \multirow{2}{*}{ Groups } & \multicolumn{2}{|c|}{ Baseline } & \multicolumn{2}{|c|}{9 Months } & \multicolumn{2}{|c|}{12 Months } \\
\hline & $\mathrm{r}$ & $\mathrm{p}$ & $\mathrm{r}$ & $\mathrm{p}$ & $\mathrm{r}$ & $\mathrm{p}$ \\
\hline $\begin{array}{c}\text { Group I } \\
\text { Cavities prepared by conventional drill } \\
\text { method. }\end{array}$ & ------- & ------- & 0.400 & 0.081 & 0.400 & 0.081 \\
\hline $\begin{array}{c}\text { Group II } \\
\text { Cavities prepared by hydro-abrasion } \\
\text { method }\end{array}$ & ------- & ------ & 0.577 & $0.008 *$ & 0.577 & $0.008 *$ \\
\hline
\end{tabular}




\section{DISCUSSION}

Dental caries is a disease of dental hard tissues, being the most common chronic disease amongst all oral conditions. It has been related to multifactorial etiology, where diet and oral flora play an essential role, being the acid production by microbial metabolism of sugars and the prolonged periods of low oral $\mathrm{pH}$, the critical factors for tooth demineralization ${ }^{17}$.

Conventional cavity preparation and caries removal methods use mechanical means, mostly burs, and are often associated with pain and fear. Although the pain can be managed through local anesthesia, fear of the needle, noise, and vibration of mechanical preparation remains a cause of discomfort for the patient. Moreover, these techniques present the risk of easily removing healthy dental tissues or damaging the pulp through temperature rise, which may be the origin of discomfort ${ }^{18}$.

Among the alternative methods of caries removal, air- abrasion may be preferable, allowing more conservative treatments, being more selective in removing decayed tissue and preserving more healthy tissue despite of prolonged treatment times. However, high speed burs yielded preparations 1.5 times quicker than air abrasion techniques ${ }^{19}$.

A combination between tactile and advanced visual technology provides greater opportunity to confirm the diagnosis of carious lesions requiring medical intervention. Therefore, ICDAS combined with DIAGNOcam have been used currently for occlusal caries detection ${ }^{20}$.

In the current study the use of rubber dam isolation was a must during cavity preparation to avoid the splattering of the powder particles from air abrasion unit within the oral cavity and/or their accidental ingestion. Additionally, patients and the operator were equipped with protective eyewear to prevent the abrasive particles from accidentally entering the eyes. In addition to the well-known benefits of using a rubber dam during the restorative procedures $^{21}$.
Comparing the conventional drill versus the hydro-air abrasion methods of cavity preparation used in the present study, the dentin surface prepared with rotating mechanical burs was reported to provide a homogeneous surface, entirely covered by smear layer and also dentinal tubules with dental plugs. While air abrasion produces a more irregular, imbricate surface pattern and a thinner smear layer when compared to bur-cut dentin ${ }^{22}$.

The use of the self-etch adhesive in the current study was related to its simplicity of use by eliminating the need for acid conditioning, rinsing, and drying of etched dental substrate and to the theoretical ability to etch and infiltrate simultaneously, thus preventing discrepancies between demineralization and infiltration and less technique sensitivity compared to etch-and-rinse adhesives ${ }^{23}$.

Reproducing occlusal anatomy using 'Stamp technique' was found to provide a precise occlusal topography, that's why it was utilized in this study ${ }^{24}$.

The present study was double-blinded in order to eliminate investigator or patient-related bias. For clinical evaluation, modified USPHS criteria were used for clinical evaluation of the restoration retention, marginal discoloration, marginal adaptation, secondary caries, and postoperative sensitivity. These criteria would be affected by the method of cavity preparation and are considered valid criteria for comparison purposes among studies at different observation periods ${ }^{25}$.

Follow up was scheduled to be at base line, 6 \& 12 months however, due to the current COVID-19 pandemic disease, it was impossible to recall the patients at 6 months follow up period and they were available only at the $9^{\text {th }}$ month. Although the study period (one year) is not considered an indication for the long term suitability of the tested materials however this testing period is acceptable and comparable with different clinical studies ${ }^{26}$.

Concerning the study results regarding the anxiety and operative pain (VAS values) that were 
recorded during cavity preparation. It was found that patients in group I felt pain rated from mild to very severe pain. However, in group II the majority of cases recorded no pain and less recorded mild pain. This corresponded with previous study in which many patients treated with conventional method requested anesthesia than those treated with air abrasion ${ }^{27}$.

Similar data have been presented in the study of Anwar et al. ${ }^{28}$ where the unpleasant sensation of scraping the decay with hand excavation and the vibration associated with the use of conventional method during caries removal makes the treatment more traumatic than air abrasion. As the rotating bur easily cuts through carious dentin to eventually open up healthy tubules deeper in the tissue and in conjunction with water stimulation of odontoblastic processes that will result in pain associated with cavity preparation using this technique.

The success of a restorative material is indicated by its longevity in the oral cavity; as such, retention rates represent the most important evaluation criteria. A high retention rate $(100 \%$ alpha scores) of the current restorations was recorded throughout the study time in the two groups. This may due to placing the restorations in small Class I cavities where no heavy occlusal loads are expected to occur, as most of the functional stresses are absorbed by the remaining tooth structure. These findings came in agreement with other ${ }^{29}$.

Concerning the clinical assessment of marginal adaptation, all tested restorations were further evaluated by SEM examination of representative inverse replica of the restorations. A satisfactory clinical performance was reported after 12 months of evaluation where the two groups recorded $100 \%$ Alpha scores denoting good adaptation. In addition, a non-statistically significant difference was recorded neither between the two groups denoting no significant effect of the cavity preparation nor among the three follow up periods, confirming that the study period did not significantly affect the marginal adaptation.

This clinical result came in agreement with Bayraktar et al. ${ }^{30}$ who demonstrated that prepared cavities with rotating bur and restored by other composite materials exhibited satisfactory marginal adaptation after 12-month clinical service.

The inverse replica has been recommended in previous study evaluate marginal and internal gaps under SEM. This was utilized in this study because it is straight forward, accurate, reliable, less costly, noninvasive, and can be repeated quickly without loss of precision. In addition, light-body vinyl polyether silicone impression material was chosen because it demonstrated excellent dimensional stability. However, a reported disadvantage of this technique is that it is a 2 -dimensionalebased method ${ }^{31}$.

The current clinical results regarding the marginal adaptation were nearly comparable to those observed by SEM examination of the inverse replica, where the latter revealed minute gaps which cannot be detected clinically. After 9, 12 months a mean value gap width in group I (cavities prepared by conventional drill method) was greater than that was recorded for group II (cavities (prepared by hydro-abrasion unit).

It was reported that the inverse replica is a sensitive, straight forward, accurate, reliable technique and any minor defects can affect the assessment of marginal integrity. This might explain the positive correlation between the SEM findings of the inverse replica and the clinical findings ${ }^{32}$.

These results came in agreement with Yazici et.al ${ }^{33}$ who compared mechanical drill method prepared cavities with those of air abraded and found that marginal seal recorded different results after different recalls ( 6 and 24 months) explained that by decreasing the integrity of the adhesive bond at the tooth/resin interface by time.

On the other hand, the current results disagree with Freeman.et al ${ }^{34}$ who studied the effect of air 
abrasion on the adaptation and shear bond strength of composite resin bonded to dentin using etchand-rinse and self-etch resin adhesives. Confocal microscopy showed both adhesives closely adapted to dentin and a significantly greater number of resin tags were observed for the etch-and-rinse adhesive. Air abrasion significantly increased resin tag length for the etch-and-rinse adhesive and significantly increased the number, length and thickness of tags for the self-etch adhesive. However, it resulted in defect formation within the hybrid layer caused separation of the hybrid layer from adjacent dentin containing resin tags after one year.

Despite the improvements in materials and techniques, postoperative sensitivity following composite restoration still remains a problem, especially in posterior teeth. Closely connected with the fact that light-cured composites undergo polymerization shrinkage which will cause cusp deflection. The incidence of post-operative sensitivity is more frequently reported for Class I composite restorations due to the configuration factor or $\mathrm{C}$-factor responsible for the stresses and poor adaptation of composite material to internal walls ${ }^{35}$.

In addition postoperative sensitivity might be related to the type of adhesive used. Findings of existing evaluations of self-etching adhesives was explained by the incorporation of the smear layer, resin monomer, collagen, and minerals into the superficial portion of the tooth surface after using self-etch adhesives. This may prevent postoperative sensitivity, which was reported to be more common after using etch-and-rinse adhesive systems ${ }^{36}$.

Observing the marginal discoloration which is the first clinical signs of the failure of composite restoration. It was currently found that the two groups revealed (100\%) Alpha scores (denoting no discoloration after 24 hours. At nine months and 12 months group I (cavities prepared by conventional drill method) Bravo scores were recorded in (20\%) and Charlie scores (15\%). While in group II (cavities prepared by hydro-abrasion unit) Bravo scores were recorded in (5\%) and (10\%) Charlie scores. The relatively low incidence of Bravo, Charlie scores for the two groups may be attributed to absence of acid etching and degradation of adhesive system at the tooth/restoration interface resulting in staining by oral fluid penetration ${ }^{37}$.

This finding came in an agreement with Galafassi et al ${ }^{38}$ who believed that the polymerization shrinkage stress of resin-composite material is the factor responsible for the evident marginal discoloration, in the restorations after 12 months.

Currently, there was a positive correlation between marginal adaptation versus marginal discoloration. These results came in agreement with and Baracco et al ${ }^{39}$ reporting that stain accumulation is associated with a margin defect resulting in marginal staining or penetration into the interface, demonstrating more of a shadow or undermining effect.

Secondary caries was not currently recorded in the restorations of both tested groups throughout the study period. The results observed after the 1 year period could provide some useful information about the clinical performance of resin materials; however, the period tested was too short to identify the development of any secondary caries. This finding was in line with other clinical study ${ }^{40}$.

Absence of secondary caries also came in agreement with Stefanski et al ${ }^{41}$ where no secondary caries was observed contiguous to the evaluated nano-filled restorations, despite the frequency of high caries risk in the participants, which could be indicative of a good marginal seal.

\section{CONCLUSION}

Hydro-abrasion could be recommended as a preferred method of cavity preparation in shallow to mild carious lesions, owing to the recorded results of being painless procedure, provided better marginal seal and so predicted longevity of composite restoration compared to the conventional cavity preparation method. 


\section{REFERENCES}

1- Dobrzański LA, Dobrzański LB, Dobrzańska-Danikiewicz AD, Dobrzańska J. The Concept of Sustainable Development of Modern Dentistry. Processes. 2020; 8(12):1-86.

2- Souza JF, Boldieri T, Diniz MB, Rodrigues JA, Lussi A, Cordeiro RD. Traditional and novel methods for occlusal caries detection. Performance on primary teeth. Lasers in Med Sci.2013;28(1):287-295.

3- Mazur M, Jedliński M, Ndokaj A, Corridore D, Maruotti A, Ottolenghi L, et al. Diagnostic Drama. Use of ICDAS II and Fluorescence-Based Intraoral Camera in Early Occlusal Caries Detection: A Clinical Study. Int. J. Environ. Res. Public Health.2020;17(8):2937.

4- Elhennawy K, Askar H, Jost-Brinkmann PG, Reda S, Al-Abdi A, Paris S, et al. In vitro performance of the DIAGNOcam for detecting proximal carious lesions adjacent to composite restorations. J Dent. 2018;72:39-43.

5- Mirsiaghi F, Leung A, Fine P, Blizard R, Louca C. An investigation of general dental practitioners' understanding and perceptions of minimally invasive dentistry. Br Dent J. 2018;225(5):420-424.

6- Anja B, Walter D, Nicoletta C, Marco F, Pezelj Ribarić S, Ivana M. Influence of air abrasion and sonic technique on microtensile bond strength of one-step self-etch adhesive on human dentin. The Sci World J. 2015;2015:1-7

7- Farooq I, Moheet IA, Alshwaimi E. Cavity cutting efficiency of a Bioglass TM and alumina powder combination utilized in an air abrasion system. Bulletin of Mat Sci. 2016;39(6):1531-1536.

8- OAESE M, Ibrahim ST, Alamoudi NM. Resin infiltration of non-cavitated proximal caries lesions: a literature review. J Oral Hyg Health. 2018;6(1):235-243.

9- Saxena C, Kartikay S, Bhakhar V, Videa M, Ghanchi M, Jani D. An Overview of Enamel Matrix Proteins. Int J Prev Clinic Dent Res. 2016; 3(1):79-84

10- Lynch RJ. The primary and mixed dentition, post-eruptive enamel maturation and dental caries: a review. Int Dent J.2013;63:3-13.

11- Abou Neel EA, Aljabo A, Strange A, Ibrahim S, Coathup M, Young AM, Bozec L, Mudera V. Demineralizationremineralization dynamics in teeth and bone. Int. J. nanomedicine. 2016;11:4743.

12- Izadi Z, Derakhshankhah H, Alaei L, Karkazis E, Jafari S, Tayebi L. Recent Advances in Nanodentistry. In
Applications of Biomedical Engineering in Dentistry. Springer, Cham. 2020; 263-287.

13- Rodas-Junco BA, Canul-Chan M, Rojas-Herrera RA, De-la-Peña C, Nic-Can GI. Stem cells from dental pulp: what epigenetics can do with your tooth? Frontiers in Physiology. 2017;8:999.

14- Pitts NB, Zero D. White paper on dental caries prevention and management. FDI World Dental Federation. 2016:3-9.

15- Melo M, Pascual A, Camps I, Del Campo Á. In vivo study of different methods for diagnosing pit and fissure caries. $\mathrm{J}$ Clinic Experiment Dent. 2015;7(3): 387.

16- Garg A, Biswas G, Saha S. Recent Advancements in Diagnosis of Dental Caries. LAP LAMBERT Academic Publishing; 2014.

17- Hon L, Mohamed A, Lynch E. Reliability of color and hardness clinical examinations in detecting dentine caries severity: a systematic review and meta-analysis. Scientific Reports. 2019;9(1):1-11.

18- Sarmadi R, Andersson EV, Lingström P, Gabre P. A Randomized Controlled Trial Comparing Er: YAG Laser and Rotary Bur in the Excavation of Caries-Patients' Experiences and the Quality of Composite Restoration. The open Dent J. 2018;12:443-454.

19- Cardoso M, Coelho A, Lima R, Amaro I, Paula A, Marto CM, Sousa J, Spagnuolo G, Marques Ferreira M, Carrilho E. Efficacy and Patient's Acceptance of Alternative Methods for Caries Removal. A Systematic Review. J Clinic Med. 2020;9(11):1-26.

20- Alaa E, El-Messiry H. Comparative Evaluation of ICDAS, WHO and Histological Examination in Detection of Occlusal Carious Lesions. Braz Dent Sci. 2020;23(3):1-8.

21- Tuncer D, Çelik Ç, Yamanel K, Arhun N. Clinical evaluation of microhybrid composites in noncarious cervical lesions: 24-month results. Niger J Clin Pract. 2017;20(2):176-181.

22- Saikaew P, Matsumoto M, Sattabanasuk V, Harnirattisai C, Carvalho RM, Sano H. Ultra-morphological characteristics of dentin surfaces after different preparations and treatments. Eur J Oral Sci. 2020;128(3):246-254.

23- Oglakci B, Arhun N. The shear bond strength of repaired high-viscosity bulk-fill resin composites with different adhesive systems and resin composite types. J Adhes Sci Tech. 2019;33(14):1584-1597. 
24- Ramseyer ST, Helbling C, Lussi A. Posterior vertical bite reconstructions of erosively worn dentitions and the "stamp technique"-A case series with a mean observation time of 40 months. J Adhes Dent. 2015;17(3):283-289.

25- Bhadra D, Shah NC, Rao AS, Dedania MS, Bajpai N. A 1-year comparative evaluation of clinical performance of nanohybrid composite with Activa ${ }^{\mathrm{TM}}$ bioactive composite in Class II carious lesion: A randomized control study. J Conservative Dent. 2019;22(1):92-96.

26- VELO MM, COELHO LV, BASTING RT, AMARAL FL, FRANÇA FM. Longevity of restorations in direct composite resin: Literature review. RGO-Revista Gaúcha de Odontologia. 2016;64(3):320-326.

27- Lai G, Lara Capi C, Cocco F, Cagetti MG, Lingström P, Almhöjd U, Campus G. Comparison of Carisolv system vs traditional rotating instruments for caries removal in the primary dentition: A systematic review and meta-analysis. Acta Odontologica Scandinavica. 2015;73(8):569-580.

28- Anwar AS, Kumar RK, Rao VA, Reddy NV, Reshma VJ. Evaluation of microhardness of residual dentin in primary molars following caries removal with conventional and chemomechanical techniques: An in vitro study. J Pharm bio allied Sci. 2017;9(1): 166-172.

29- Shaalan OO, Abou-Auf E, El Zoghby AF. Clinical evaluation of self-adhering flowable composite versus conventional flowable composite in conservative Class I cavities: Randomized controlled trial. J Conservative Dent: 2018;21(5):485-490

30- Bayraktar Y, Ercan E, Hamidi MM, Çolak H. One-year clinical evaluation of different types of bulk-fill composites. J Inves Clin Dent. 2017;8(2):1-9.

31- Schönberger J, Erdelt KJ, Bäumer D, Beuer F. Evaluation of two protocols to measure the accuracy of fixed dental prostheses: An in vitro study. J Pros. 2019;28(2): 599-603.

32- Heintze SD. Clinical relevance of tests on bond strength, microleakage and marginal adaptation. Dent Mater. 2013; 29(1):59-84.

33- Yazici AR, Antonson SA, Kutuk ZB, Ergin ES. Thirty-sixmonth clinical comparison of bulk fill and nanofill composite restorations. Oper Dent. 2017;42(5):478-785.

34- Freeman R, Varanasi S, Meyers IA, Symons AL. Effect of air abrasion and thermocycling on resin adaptation and shear bond strength to dentin for an etch-and-rinse and self-etch resin adhesive. Dent Mater. 2012;30(2):180-188.

35- Sabbagh J, Fahd JC, McConnell RJ. Post-operative sensitivity and posterior composite resin restorations: a review. Dental Update. 2018;45(3):207-213.

36- Kurokawa H, Takamizawa T, Rikuta A, Tsubota K, Miyazaki M. Three-year clinical evaluation of posterior composite restorations placed with a single-step self-etch adhesive. J Oral Sci. 2015;57(2):101-108.

37- Çelik Ç, Arhun N, Yamanel K. Clinical evaluation of resinbased composites in posterior restorations: a 3-year study. Medical Principles and Practice. 2014;23(5):453-459.

38- Galafassi D, Scatena C, Galo R, Curylofo-Zotti FA, Corona SA, Borsatto MC. Clinical evaluation of composite restorations in Er: YAG laser-prepared cavities re-wetting with chlorhexidine. Clin Oral Investig. 2017;21(4):1231-1241.

39- Baracco B, Perdigão J, Cabrera E, Giráldez I, Ceballos L. Clinical evaluation of a low-shrinkage composite in posterior restorations: one-year results. Oper Dent. 2012;37(2):117-129.

40- Torres CR, Rêgo HM, Perote LC, Santos LF, Kamozaki $\mathrm{MB}$, Gutierrez NC, et al. A split-mouth randomized clinical trial of conventional and heavy flowable composites in class II restorations. J Dent. 2014;42(7):793-799.

41- Stefanski S, van Dijken JW. Clinical performance of a nanofilled resin composite with and without an intermediary layer of flowable composite: a 2-year evaluation. Clin Oral Investig. 2012;16(1):147-153. 